Hydroécol. Appl. (1992) Tome 4 Vol 2, pp. 23-31

\title{
Simulation mathématique des nappes d'hydrocarbures et comparaison avec les observations par télédétection
}

\section{Mathematical simulation of oil spill at sea and comparison with observations by remote sensing}

\author{
K. Maroihi \\ Geohydrodynamics and Environmental Research, (GHER), University of Liège, B5, Sart Tilman, \\ $B-4000$ Liège, Belgium

\section{E. Deleersnijder} \\ Institute of Astronomy and Geophysics, Catholic University of Louvain, 2, chemin du Cyclotron, \\ B-1348 Louvain-la-Neuve, Belgium
}

\section{A. Loffet}

Walphot Detection S.A., rue Van Opré 97, 5100 Namur, Belgium

Résumé. - On présente un modèle mathématique du transport et de l'étalement des nappes d'hydrocarbures à la surface de la mer.

Le modèle se distingue des modèles antérieurs par sa capacité à prendre en compte simultanément les effets de gravité, friction, et de tension superficielle, - permettant ainsi la modélisation des nappes expérimentales de petites dimensions comme des grandes nappes accidentelles -, et par une nouvelle paramétrisation de la tension superficielle et du frottement interfacial, mieux adaptée aux conditions réelles en mer.

La performance du modèle est évaluée par la modélisation d'expériences in situ où la position et l'épaisseur de la nappe sont mesurées par télédétection. Un excellent accord est trouvé entre les prévisions du modèle et les observations.

Abstract. - A mathematical model of oil spill's transport and spreading is presented. The model differs from previous ones by its capability of taking simultaneously gravity, friction and surface tension into account - allowing the simulation of small experimental spills as well as large accidental spills - and by a new parameterization of surface 
tension and interface stresses, better adapted to real field conditions.

The model is tested by the simulation of in situ experiments where the position and the thickness of the spill are measured by remote sensing. An excellent agreement is found between the model's predictions and the observations.

\section{INTRODUCTION}

Le développement intensif des forages "off-shore" et le grand trafic des tankers ont augmenté les risques de pollution par hydrocarbures.

L'importance des dommages écologiques et économiques causés par les nappes d'hydrocarbures, déversées accidentellement ou volontairement, sont tels qu'ils ont suscité un vaste effort international dans l'amélioration et le perfectionnement des techniques de protection et de nettoyage et dans l'implantation des systèmes de détection et de pistage.

La télédétection peut jouer un rôle majeur dans les problèmes de pollution par les hydrocarbures. Ce rôle consiste en deux points:

1) La recherche et la localisation des régions polluées. Cette tâche est principalement une tâche de surveillance incluant la détection des opérations de décharges illégales par les navires passants, leur identification et la fourniture de preuves aux tribunaux.

2) La cartographie et le pistage de la nappe et la classification par épaisseur des hydrocarbures en vue d'assister les opérations de nettoyage.
La modélisation du transport et de l'étalement des nappes d'hydrocarbures est très difficile. Elle requiert une bonne connaissance des conditions météorologiques et océanographiques et des caractéristiques physico-chimiques des hydrocarbures.

Le présent modèle est capable d'évaluer le transport général sous l'action combinée du vent et des courants, et son étalement progressif sous l'action combinée de la gravité, de la tension superficielle et de la friction. L'évaporation, l'entraînement des hydrocarbures dans la colonne d'eau et de l'eau dans la nappe peuvent être tenus en compte.

On peut synthétiser le modèle en une équation d'évolution de l'épaisseur de la nappe d'hydrocarbures. Ce paramètre contient les caractéristiques essentielles du phénomène et présente l'avantage d'utiliser les données de la télédétection.

\section{LE TRANSPORT}

À la surface de l'eau, une nappe d'hydrocarbures a tendance à dériver sous l'action combinée du vent et des courants marins (marées, tempêtes, 
circulation résiduelle...). Ainsi, le vecteur vitesse de dérive du centre de masse de la nappe est donné par:

$$
\vec{V}_{d}=\dot{V}_{v}+\dot{V}_{c}
$$

où $V_{v}$ est le vecteur vitesse de dérive dû au vent et $\vec{V}_{c}$ celui dû aux courants marins. L'équation du mouvement du centre de masse s'écrit alors:

$$
\frac{d \dot{X}_{c}}{d t}=\vec{V}_{v}+\dot{V}_{c}
$$

$\vec{V}_{v}$ est dévié à droite de la direction du vent d'un angle $\alpha$ (dérive d'Ekman) et sa grandeur peut être reliée au vent à $10 \mathrm{~m}$ de la surface par des relations semi-empiriques (Nihoul, 1983). L'angle de dérive peut être relié à l'intensité du vent et à la latitude.

Les courants marins sont déterminés par les observations ou par des modèles hydrodynamiques régionaux.

\section{L'ÉTALEMENT}

Fay et Nihoul ont développé chacun un modèle de l'étalement d'une nappe d'hydrocarbures axi-symétrique.

L'étalement se décompose en trois régimes:

1) Inertie-gravité.

2) Gravité-friction.

3) Friction-tension superficielle.

Dans chacun des régimes, une relation entre la surface de la nappe et le temps, basée sur une étude dimensionnelle, a été donnée.

La paramétrisation de la friction utilisée par Fay dans son modèle fait in- tervenir la viscosité moléculaire. Pour cette raison, ce modèle n'est apte à décrire que des expériences en laboratoire ou en mer très calme.

La paramétrisation utilisée dans le modèle de Nihoul est identique à celle proposée par Warluzel et Benque (1981). Elle est la mieux adaptée aux conditions réelles en mer (Nihoul, 1983, 1984).

Dans le modèle de Nihoul (ce modèle sera décrit brièvement par la suite), la surface (S) de la nappe est reliée au temps (t) par les relations suivantes (Nihoul, 1983):

Régime inertie-gravité

$$
S \sim \mathrm{g}^{1 / 2} \Omega^{1 / 2} \mathrm{t}
$$

Régime gravité-friction

$$
S \sim g^{1 / 3} \Omega^{2 / 3} k^{-1 / 3} \rho^{1 / 3} t^{1 / 3}
$$

Régime friction-tension superficielle

$$
\mathrm{S} \sim \gamma \mathrm{k}{ }^{1} \mathrm{t}
$$

$\Omega$ est le volume déversé, $\mathrm{k}$ le coefficient de friction à l'interface entre les hydrocarbures et l'eau, $\gamma$ la tension superficielle, $\rho$ la densité des hydrocarbures et

$$
g^{\prime}=\frac{\rho^{w}-}{\rho^{w}} \rho \sim 1
$$

$\rho^{w}$ est la densité de l'eau.

Les temps auxquels se produisent les transitions d'un régime à l'autre s'obtiennent en égalisant les relations (3) et (4) d'une part et (4) et (5) d'autre part. Ainsi:

Régime inertie-gravité $\rightarrow$ Régime gravité-friction

$$
t_{\alpha}=\Omega^{1 / 4} g^{\prime-1 / 4} k^{-1 / 2} \rho^{1 / 2}
$$


Régime inertie-gravité $\rightarrow$ régime friction-tension superficielle

$$
t_{y}=\Omega \mathrm{g}^{1 / 2} \gamma^{3 / 2} k \rho^{1 / 2}
$$

La figure 1 montre les phases successives de l'étalement pour différents volumes déversés.

La paramétrisation de la friction adoptée conduit à des lois de l'étalement qui diffèrent de celles données par Fay (1969), mais qui sont en excellent accord avec les observations.

\section{LE MODĖLE}

Le modèle a été décrit dans une publication antérieure (Nihoul, 1983). Les équations du modèle intégrées sur l'é- paisseur de la nappe peuvent être écrites (Nihoul, 1983):

$$
\begin{aligned}
& \frac{\partial(h \cup ̉)}{\partial t}+\nabla \cdot(h \cup ́ u ́)= \\
& -h \nabla\left(\begin{array}{c}
p_{a} \\
\rho
\end{array}+g^{\prime} h\right)+\left(\tau_{\delta}-\tau^{b}\right) \rho 1 \\
& \frac{\partial h}{\partial t}+\vec{\nabla} \cdot(h \bar{U})=-q
\end{aligned}
$$

où $h$ est l'épaisseur de la nappe, $\vec{U}$ la vitesse horizontale moyenne sur la nappe, $\mathrm{P}_{\mathrm{a}}$ la pression atmosphérique (supposée constante sur la région de la nappe, $\tau_{0}^{\mathrm{s}}\left(\tau_{0}^{\mathrm{b}}\right)$ la tension de surface dans la nappe à l'interface de celle-ci et de l'air (de l'eau), q est le taux de variation de l'épaisseur de la nappe par évaporation, entraînement des

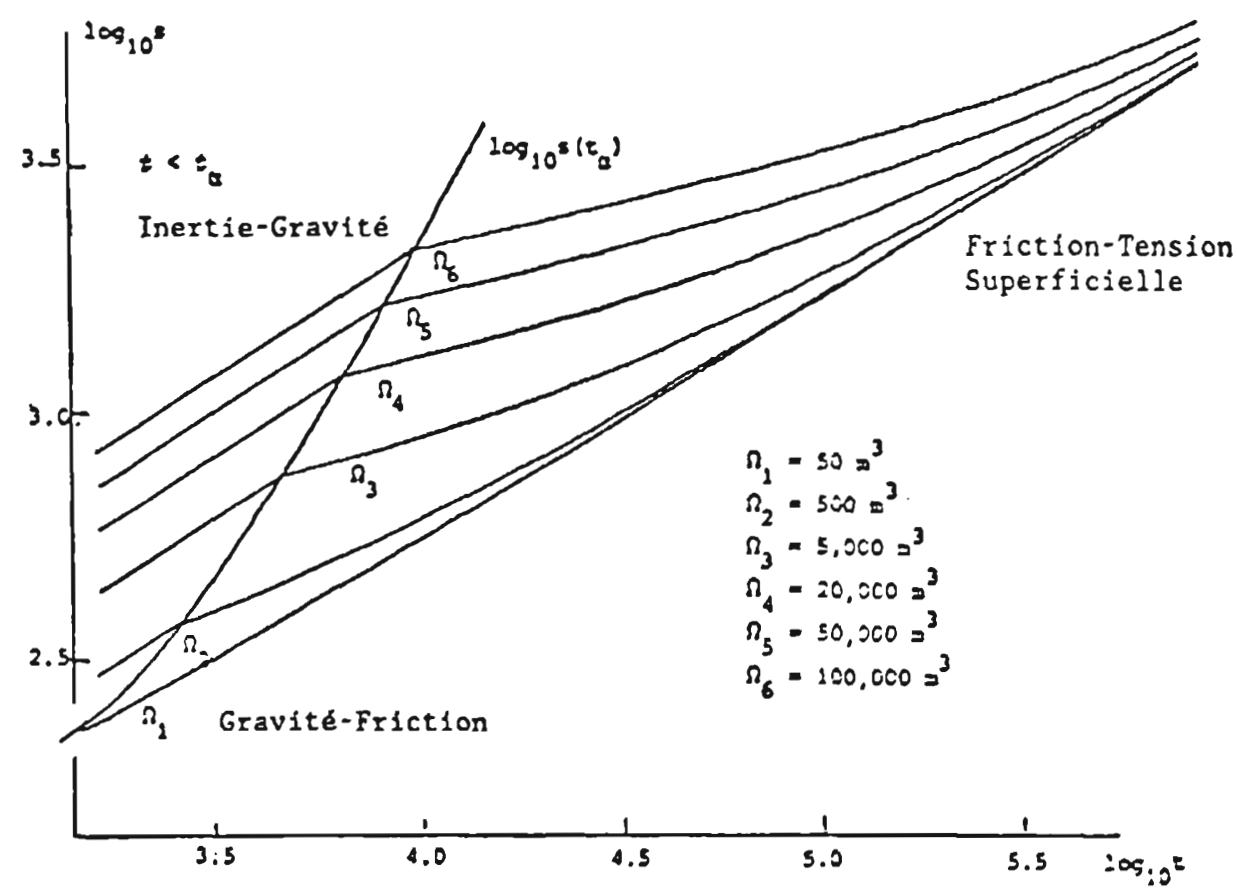

Fig. 1. Variation du rayon de la nappe en fonction du temps pour différents volumes déversés. 
hydrocarbures dans la colonne d'eau et de l'eau de mer dans la nappe (on supposera $q \geq 0$, et se plaçant dans le cas le plus défavorable, on prendra $q=0), \rho, \rho^{w}$ et $g^{\prime}$ ont été défini précédemment.

La force normale résultant de la tension superficielle est négligée mais la force tangentielle est prise en compte. Ainsi (Nihoul, 1983):

$$
\begin{aligned}
\vec{\tau}-\vec{\tau}= & \vec{\tau}_{a}^{s}-k\left(\vec{U}-U^{j w}\right) \\
& +\left(\gamma_{w a}-\gamma_{0 a}^{0}-\gamma_{0 w}^{0}\right) \vec{\nabla} ø
\end{aligned}
$$

où $\tau_{a}^{s}$ est la tension due au vent, $\mathrm{k}$ le coefficient de friction (à l'interface déformable et perméable entre les hydrocarbures et l'eau, on adopte une représentation linéaire de la tension $\tau_{0}^{b}$, en fonction de la vitesse moyenne Ú, e.g. Pedersen, 1972), Üw la vitesse du courant sous la nappe, $\gamma_{w a}$ la tension superficielle à l'interface eau-air, $\gamma_{0 a}\left(\gamma_{0 w}\right)$ la tension superficielle à l'interface hydrocarbures-air (hydrocarbures-eau), $\varnothing$ une fonction traduisant les variations des tensions superficielles en fonction du mélange progressif des hydrocarbures et de l'eau de leurs valeurs $\gamma_{0 a}^{0}$ et $\gamma_{0 w}^{0}$ au centre de la nappe (hydrocarbures purs), à leurs valeurs $\gamma_{w a}$ et $O$ au bord de celle-ci.

Le régime inertial est relativement court et ne concerne que les premiers instants de l'étalement. En le négligeant et en combinant les équations (9) et (10), on obtient compte tenu de l'équation (12):

$$
\begin{array}{r}
\frac{\partial h}{\partial t}+\vec{\nabla} \cdot\left(\left(U^{j a}+U^{j c}+\mu \vec{\nabla} ø\right) h\right) \\
=\lambda \vec{\nabla} \cdot\left(h^{2} \vec{\nabla} h\right)
\end{array}
$$

où $\vec{U}_{a}=\vec{\tau}_{a}^{s} / k$ (14) est la vitesse d'advection due au vent et à l'action des courants induits localement par celuici, $\vec{U}^{w}$ la vitesse du courant marin régional (marée, tempête, circulation résiduelle),

$$
\begin{gathered}
\mu=\left(\gamma_{w a}-\gamma_{a}-\gamma_{w a}^{0}\right) / k \\
\lambda=g^{\prime} \rho k^{-1}
\end{gathered}
$$

Le transport dû au vent est paramétrisé, d'après les observations, comme suit (Nihoul, 1983):

$$
\left\|\vec{U}^{a}\right\|=10^{-3 / 2}\|\vec{V}\|
$$

où $V$ est la vitesse du vent à $10 \mathrm{~m}$ de la surface. Le vecteur $\vec{U}^{a}$ est dévié à droite de $V$ d'un angle:

$$
\begin{aligned}
& \alpha=40-8\|\vec{V}\|^{1 / 2} \\
& \text { pour } 0 \leq\left\|V^{\prime}\right\|<25 \mathrm{~m} / \mathrm{s} \\
& \alpha=0 \text { pour }\|\vec{V}\|>25 \mathrm{~m} / \mathrm{s}
\end{aligned}
$$

Pour les expériences en Mer du Nord, les courants marins sont déterminés à partir du modèle hydrodynamique du GHER.

L'effet des forces de tensions superticielles est relié aux gradients de tensions superficielles. Ces derniers résultent de l'entraînement de l'eau de mer dans la nappe et produisent un processus d'advection des hydrocarbures qui se superpose à celui associé aux vents et aux courants. En admettant que la nappe répond élastiquement à la tension superficielle (Batchelor 1967, Chia-Shun Yih 1968, Nihoul 1983), la forme appropriée pour $\varnothing$ est alors (Nihoul 1983, 1984):

$$
\emptyset=\frac{r^{2}}{s^{2}}
$$


où r est la distance au centre de la nappe et $s$ son rayon à l'instant $t$ défini par

$$
\mathrm{S}=\pi \mathrm{s}^{2}
$$

$S$ est la surface de la nappe à linstant $t$.

Dans des conditions de courant et de vent relativement calmes, l'advection associée au vent et aux courants est spatialement constante, et dans un système d'axes associé au centre de la nappe, on peut supposer en première approximation, une symétrie cylindrique de cette dernière. L'équation (13) s'écrit alors en coordonnées polaires:

$\frac{\partial h}{\partial t}+\frac{1}{r} \frac{\partial}{\partial r}\left(2 \mu \stackrel{r^{2}}{s^{2}} h\right)=\frac{1}{r} \frac{\partial}{\partial r}\left(\lambda r h^{2} \frac{\partial h}{\partial r}\right)$

Cette équation admet des solutions analytiques de la forme:

$$
h=B^{-2} f(\eta)
$$

où $\eta=r / s$

et où $B$ est une constante qui sera déterminée par la suite. La fonction $f$ doit satisfaire à la condition:

$$
f(1)=0
$$

En substituant la fonction (22) dans l'équation (21), on obtient deux équations différentielles pour $\int$ et $\mathrm{s}$ :

$$
\begin{aligned}
& \frac{d}{d t}\left(\eta f^{2} \frac{d f}{d \eta}+\eta^{2} f\right)=0 \\
& s \frac{d s}{d t}-\lambda B^{2} s^{-4}=2 \mu
\end{aligned}
$$

Tenant compte de la condition (24), l'équation (25) donne:

$$
f(\eta)=\left(1-\eta^{2}\right)^{1 / 2}
$$

La valeur de $B$ est déduite de la condition de constance de volume, $\mathrm{q}$ étant nul, l'équation (10) du modèle se réduit à:

$$
\int_{S} h d S=\Omega \quad \forall t
$$

où $\Omega$ est le volume initialement déversé. En combinant les équations (29), (28) et (23), on trouve:

$$
B=\begin{aligned}
& 3 \Omega \\
& 2 \pi
\end{aligned}
$$

L'intégration de l'équation donne:

$$
S-\frac{1}{a} \operatorname{arctg}(\mathrm{aS})=4 \pi \mu \mathrm{t}
$$

$$
\begin{aligned}
& \text { où } a=c \begin{array}{c}
2 \\
3 \Omega
\end{array}\left(\begin{array}{l}
2 \mu \mathrm{k} \\
g^{\prime} \rho
\end{array}\right)^{1 / 2} \\
& \mathrm{~S}=\pi \mathrm{s}^{2}
\end{aligned}
$$

L'équation (30) donne le rayon de la nappe en fonction du temps. Son comportement asymptotique est en parfait accord avec les prédictions de l'analyse dimensionnelle (Nihoul 1983):

$\mathrm{as}^{2}<1$ (régime gravité-friction)

$$
s-\lambda^{1 / 6} \Omega^{1 / 3} t^{1 / 6}
$$

$\mathrm{as}^{2}>1$ (régime friction-tension superficielle)

$$
s \sim 2\left(\begin{array}{l}
y \\
k
\end{array}\right)^{1 / 2} t^{1 / 2}
$$


COMPARAISON

\section{AVEC LES OBSERVATIONS}

En 1981, les valeurs observées par Kuipers lors d'une expérience de déversement au large des côtes hollandaises $\left(3 \mathrm{~m}^{3}\right)$ se disposent fidèlement sur la droite caractéristique de l'étalement dans la phase friction-tension (fig. 2).

Une série d'expériences de déversements conduites entre août 71 et août 72 au large des côtes de Virginie ont toutes mis en évidence une configuration typique: une région centrale d'épaisseur de $1 \mathrm{~mm}$ ou plus, contenant plus de $90 \%$ du volume déversé pour une surface de moins de $10 \%$, entourée d'une nappe plus vaste, d'épaisseur plus petite de plusieurs ordres de grandeurs (Hollinger et Mennella 1973). L'interprétation de ces observations suggère que l'étale-

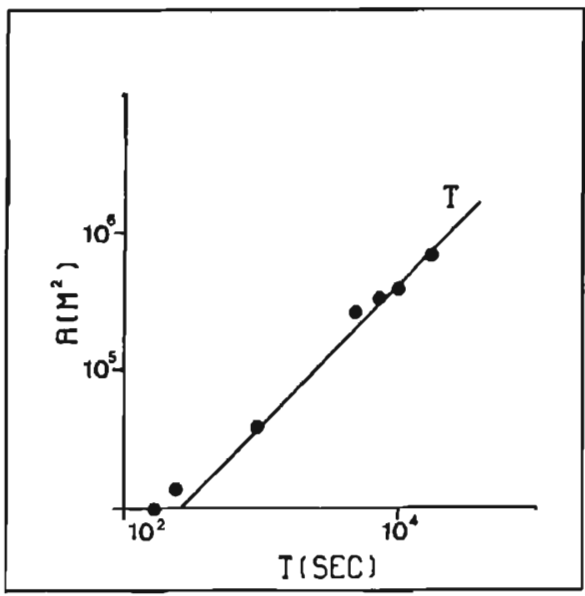

Fig. 2. Variation de la superficie de la nappe dans le cas d'un déversement expérimental de $3 \mathrm{~m}^{3}$. Comparaison entre le modèle et les observations. ment de la tache centrale est commandé par la gravité, tandis que la couche mince périphérique est rapidement sujette aux forces de tension superficielle.

Ceci est vérifié sur la figure 3 qui montre les variations temporelles des surfaces de la tache centrale (...) et de la nappe totale (xxx) observées par temps calme (vitesse du vent $2,4 \mathrm{~m} / \mathrm{s}$, Hollinger et Mennella).

Lors de l'expérience Archimèdes (oct. 21-22, 1983) conduite en Mer du Nord, les données de la télédétection n'étaient pas suffisantes pour comparer les prévisions du modèle aux observations. L'application du modèle a été limitée à l'évaluation du transport général de la nappe (fig. 4). A la fin des cinq prédictions rapportées sur la figure 4 , les écarts entre les positions calculées et celles observées se situent entre $0,33 \mathrm{~km}$ et $2,5 \mathrm{~km}$. Consi-

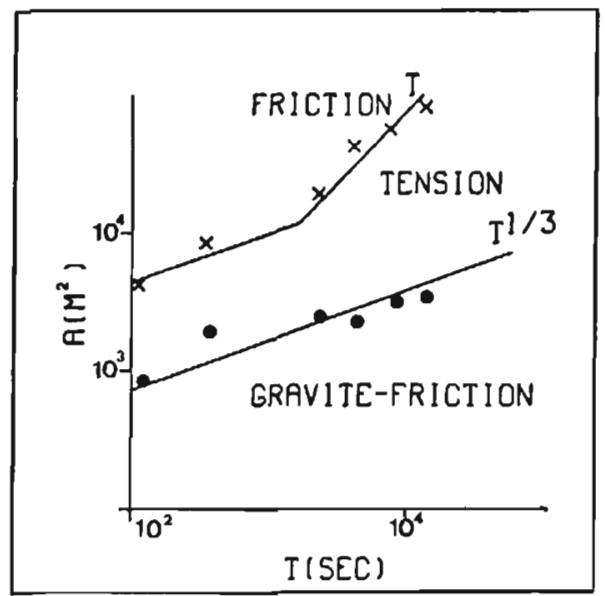

Fig. 3. Variation de la surface de la tache centrale (...) et de la surface totale de la nappe $(x x x)$ lors d'une expérience de déversement de $3.38 \mathrm{~m}^{3}$. Comparaison entre modèle ef observations. 


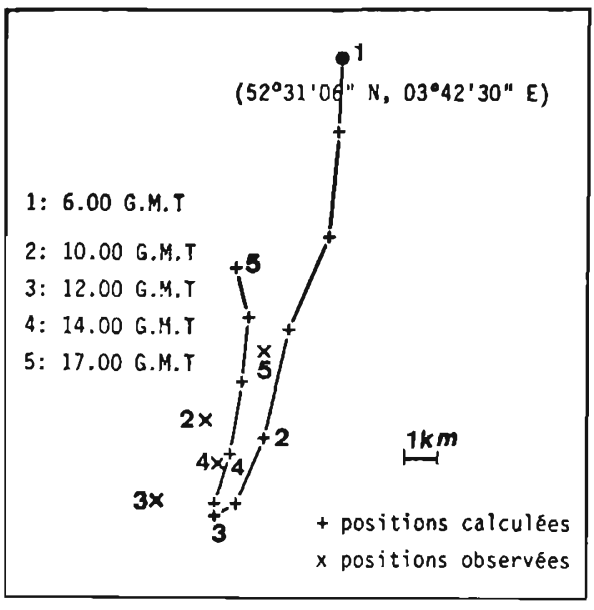

Fig. 4. Trajectoire de la nappe dans le cas de l'expérience Archimèdes. Comparaison entre le modèle et les observations.

dérant que les courants marins prédits, et non ceux localement observés, ont été utilisés dans le modèle, il est évident que les résultats concordent remarquablement avec les observations.

\section{CONCLUSION}

Le modèle présenté ci-dessus est capable de tenir compte simultanément de la gravité, de la friction, de la tension superficielle, des effets du vent et des courants.

La comparaison entre les prévisions du modèle et les résultats des expériences sus mentionnées mettent en évidence sa capacité à décrire des situations réelles en mer.

Les résultats du modèle peuvent être utilisés d'une manière dynamique dans l'interprétation, l'extrapolation et l'interpolation des données de la télédétection.

La connexion entre les modèles mathématiques et la télédétection peut jouer un rôle majeur dans l'amélioration et le renforcement des techniques de nettoyage.

\section{RÉFÉRENCES}

Batchelor G.K., 1967. An introduction to fluid dynamics. Cambridge Univ. Press, $615 \mathrm{pp}$.

Deleersnijder E., 1991. Revisting Nihoul's model for oil slicks transport and spreading on the sea. Submitted to Ecological Modelling.

Fay J.A., 1969. In: Oil on the sea, D.P. Hoult ED, Plenum Publ., New York, 53-63.

Garrett W.D. \& Barger W.R., 1970. Environ. Sec. Technol. 4: 123-127.

Gillot R.M., 1983. North Sea campaign on remote sensing of oil spill. Oct. 83, ACPH WDI, 7 pp.

Hollinger J.P. \& Mennella R.A., 1973. Measurements of the distribution and volume of sea surface oil spills using multifrequency microwave radiometry. Nat. Res. Lab., Washington, Rep., 7512, 5 pp.

Hoult D.P., 1972. Oil spreading on the sea. Ann. Rev. Fluid Mech, 4: 341-368.

Kuipers H.D., 1981. A simulation model for oil slicks at sea. North Sea Directorate, Department of Public Works, The Netherlands, $180 \mathrm{pp}$.

Lee R.A.S., 1971. A study of the surface tension controlled regime of oil spread. SM Thesis MIT.

Massin J.M. \& Vollmers R.R., 1979. Télédétection des rejets d'hydrocarbures dans les eaux côtières. Ministère de 
l'Environnement et du Cadre de Vie, Paris, $343 \mathrm{pp}$.

Nihoul J.C.J., 1983. A non-linear mathematical model for the transport and spreading of oil slicks. In: Modelling the fate and effect of toxic substances in the environment, Proc. Coll. Internat. Soc. of Ecological Modelling, June 6-10, 1983, Copenhagen.

Nihoul J.C.J., 1984. Modèle de transport et d'étalement des nappes d'hydrocarbures et comparaison avec les observations. Société Hydrotechnique de France, XVIII Journées de l'Hydraulique, Marseille, 11, 12 et 13 septembre 1984, Rapport $\mathrm{n}^{\circ} 10$ IV 10.1-IV $10.4 \mathrm{pp}$.
Pedersen F.B., 1972. Gradually varying two-layer stratified flow. Proc. Ann. Soc. Civil Eng., HYI, 98: 257-268.

Smith J.E., 1968. Torrey Canion pollution and marine life. Cambridge Univ. Press.

Venkatesh S., Neralka V.R., Jarvic E.C., Jessup R.G. et De Lorenzis B., 1981. A numerical model for predicting the movement of oil slicks. In: Mechanics of oil slicks, ENCP Ed., Paris: 219233.

Warluzel A. et Benque J.P., 1981. Un modèle mathématique de transport et d'étalement d'une nappe d'hydrocarbures. In: Mechaniscs of oil slicks, ENPC Ed., Paris: 199-211. 